\title{
Complicated Lemierre Syndrome Caused by Streptococcus gordonii and Possible Rickettsial Co-Infection in a Patient with Thrombophilia Predisposition
}

\author{
Antreas loannou $^{1}$, Dimitra Dimitriou $^{1}$, Panagiotis Dimitriou ${ }^{1}$, Aram Katsios $^{1}$, Georgios Petrikkos $^{2}$ \\ ${ }^{1}$ Internal Medicine Department, Nicosia General Hospital, 2029 Strovolos, Nicosia, Cyprus \\ ${ }^{2}$ Infectious Diseases Research Laboratory, 4th Dept. of Internal Medicine, National and Kapodistrian University of Athens, Medical School, "Attikon” \\ University Hospital, Athens, Greece
}

Received: $18 / 02 / 2017$

Accepted: $24 / 02 / 2017$

Published: $17 / 03 / 2017$ How to cite this article: Ioannou A, Dimitriou D, Dimitriou P, Katsios A, Petrikkos G. Complicated Lemierre syndrome caused by streptococcus gordonii and
possible rickettsial co-infection in a patient with thrombophilia predisposition. EJCRIM 2017;4: doi:10.12890/2017_000606.

Conflicts of Interests: The Authors declare that there are no competing interests.

This article is licensed under a Commons Attribution Non-Commercial 4.0 License

\begin{abstract}
Aims: Lemierre syndrome is a life-threating condition characterized by recent oropharyngeal infection, internal jugular vein thrombosis, and anaerobic septicemia. It is usually caused by Fusobacterium necrophorum.

Methods: A young Romanian male presented with fever and rigors, mild tachypnea, hypoxia, sore throat, decayed teeth, and tenderness of the left carotid triangle. Laboratory examination indicated severe sepsis with disseminated intravascular coagulation, acute renal failure, and acute respiratory distress syndrome while the Doppler ultrasonography of the carotids revealed left internal jugular venous thrombosis. The patient was administered piperacillin/tazobactam and vancomycin intravenously, doxycycline orally, and anti-coagulation by enoxaparin based on the diagnosis of Lemierre syndrome. Meanwhile, he was complicated by bilateral diffuse pulmonary cavities and encapsulated pleural effusions and so transcutaneous drainage was performed. The patient was discharged after a month and continued his treatment with oral phenoxypenicillin and doxycycline until full radiographic improvement. He was switched to oral anti-coagulation by vitamin-K antagonists and was referred to a hematologist, a vascular-surgeon, and a dentist.

Results: Streptococcus gordonii was isolated from the patient's blood and pleural fluid cultures and serology for Rickettsial spp. IgM was positive. Thrombophilia genetic tests revealed three minor mutations for fibrinogen-455, plasminogen activator inhibitor-1, and methylenetetrahydrofolate reductase. According to the literature, S. gordonii is not usually a causative agent and Rickettsial spp. have as yet not been correlated with Lemierre syndrome. The failure of left jugular vein recanalization shows a possible causative role of the underlying thrombophilic predisposition.

Discussion: Because of the syndrome's rarity and the atypical microorganisms isolated in this case, increased awareness is advised for its diagnosis and the underlying mechanisms involved in its genesis. The role of anti-coagulation is debatable.
\end{abstract}

\section{LEARNING POINTS}

- Very rare condition. Medical practitioners should be vigilant in its diagnosis since septic jugular vein thrombophlebitis could be misdiagnosed as neck lymphadenitis.

- Duration of antibiotic therapy and additional anticoagulation treatment are still under discussion.

- Underlying thrombophilia predisposition should be excluded. 


\section{KEYWORDS}

Septic thrombophlebitis, Lemierre, Rickettsia, thrombophilia, Streptococcus gordonii

\section{CASE REPORT}

A 23-year-old Romanian male with an unremarkable medical history was admitted after complaining about fever with rigors and a sore throat starting a week before he presented at the hospital. He was already being treated with antibiotics (amoxicillin/clavulanic acid). He recalled dust/soil exposure and a recent trip to Romania. He denied intake of alcohol and use of recreational drugs.

Physical examination revealed fever (temperature $38.1^{\circ}$ ) with rigors, tachycardia $(148 \mathrm{bpm}$ ), signs of dehydration, and a mild $2 / 6$ systolic murmur at the aortic valve position. Auscultation revealed mild crackles of the right middle and lower lobe. Tachypnea (20 breaths per minute) and hypoxia $\left(\mathrm{SaO}_{2}: 89 \%\right)$ were also present. Other findings included herpes labialis, decayed teeth in the right lower mandible, tonsillitis without exudate, and a mildly tender neck lymphadenopathy of the left carotid triangle.

Laboratory tests indicated an underlying infection causing severe sepsis and disseminated intravascular coagulation (DIC) (Table 1a). Air blood gases revealed hypoxia $\left(\mathrm{PO}_{2}: 53.8 \mathrm{mmHg}, \mathrm{SaO}_{2}: 87.7 \%\right.$ ) with mild elevation of lactic acid ( $\left.1.3 \mathrm{mmol} / \mathrm{L}\right)$. Multiple insterstitial pulmonary infiltrates and signs of acute respiratory distress syndrome (ARDS) were shown on the chest radiograph (Figure 1a).

\begin{tabular}{|l|l|l|l|}
\hline Parameter & Day 1 & Day 36 & Reference \\
\hline White blood cells (WBCs) & 26.01 & 9.52 & $\begin{array}{l}\times 10^{\wedge} 9 / \mathrm{L} \\
(3.91-8.77)\end{array}$ \\
\hline Neutrophils (\%) & $\begin{array}{l}21.57 \\
(82.8 \%)\end{array}$ & $\begin{array}{l}5.22 \\
(54.9 \%)\end{array}$ & $\begin{array}{l}\times 10^{\wedge} 9 / \mathrm{L} \\
(1.82-7.42)\end{array}$ \\
\hline Hemoglobin & 14.5 & 11.5 & $\mathrm{~g} / \mathrm{dL}(11.9-15.4)$ \\
\hline Mean Cell Volume (MCV) & 85.8 & 85.9 & $\mathrm{fL} \mathrm{(77.0-93.0)}$ \\
\hline $\begin{array}{l}\text { Mean Corpuscular } \\
\text { Haemoglobin (MCH) }\end{array}$ & 29.8 & 27.4 & $\mathrm{pg}(27.0-32.0)$ \\
\hline Reticulocytes\% & 0.2 & $4.2 \%$ & $\%(0.039-0.057)$ \\
\hline \begin{tabular}{l} 
Platelets (PLTs) \\
\hline Ratio (INR)
\end{tabular} & 19 & 308 & $\begin{array}{l}\times 10^{\wedge} 9 / \mathrm{L} \\
(150-450)\end{array}$ \\
\hline Fibrinogen & 1.43 & 1.36 & $0.95-1.02$ \\
\hline D-Dimers & 438.5 & 335.1 & $\begin{array}{l}\mathrm{mg} / \mathrm{dL} \\
(270.0-470.0)\end{array}$ \\
\hline Glucose & 11499 & & $\begin{array}{l}\mathrm{ng} / \mathrm{ml} \\
(0.0-550.0)\end{array}$ \\
\hline
\end{tabular}

\begin{tabular}{|c|c|c|c|}
\hline Parameter & Day 1 & Day 36 & Reference \\
\hline Urea & 103 & 39 & $\mathrm{mg} / \mathrm{dL}$ (17-43) \\
\hline Creatinine $(\mathrm{Cr})$ & 1.62 & 0.70 & $\mathrm{mg} / \mathrm{dL}(0.67-1.17)$ \\
\hline Proteins & 5.5 & 7.4 & $6.6-8.3 \mathrm{~g} / \mathrm{dL}$ \\
\hline Albumin & 2.4 & 4.2 & $3.5-5.2 \mathrm{~g} / \mathrm{dL}$ \\
\hline Total Bilirubin & 2.71 & 0.41 & $\mathrm{mg} / \mathrm{dL}(0.3-1.2)$ \\
\hline Direct Bilirubin & 1.22 & - & \\
\hline Alkaline phosphatase (ALP) & 413 & 90 & IU/L (30-120) \\
\hline $\begin{array}{l}\text { Gamma-Glutamyltransferase } \\
(\mathrm{y}-\mathrm{GT})\end{array}$ & 134 & 21 & IU/L (9-55) \\
\hline $\begin{array}{l}\text { Lactate degydrogenase } \\
\text { (LDH) }\end{array}$ & 684 & 399 & IU/L (208-480) \\
\hline C-reaction protein (CRP) & 293.20 & 16.36 & $\begin{array}{l}\mathrm{mg} / \mathrm{L} \\
(0.00-5.00)\end{array}$ \\
\hline Procalcitonin (PCT) & 5 & Negative & $<0.15 \mathrm{ng} / \mathrm{ml}$ \\
\hline Antistreptolysin (ASTO) & 32 & - & $10-200 \mathrm{IU} / \mathrm{ml}$ \\
\hline Lupus anticoagulant & Positive & Negative & \\
\hline Lupus anticoagulant ratio & 1.3 & Negative & $0.8-1.2$ \\
\hline
\end{tabular}

Table 1a. Laboratory examination of patient serum values during admission and after 36 days of hospitalization

\begin{tabular}{|l|l|l|l|}
\hline Parameter & Day 1 & Day 14 & Day 60 \\
\hline R. typhi IgM & $1 / 64$ (positive) & $1 / 256$ (positive) & $1 / 256$ \\
\hline R. typhi IgG & Negative & Negative & Negative \\
\hline R. conorii IgM & $1 / 64$ (positive) & $1 / 256$ (positive) & $1 / 128$ \\
\hline R. conorii IgG & Negative & Negative & Negative \\
\hline
\end{tabular}

Table 1b. Patient's serological examination for Rickettsia spp. during admission and after 60 days of hospitalization 
He was administered a broad-spectrum antibiotic therapy with intravenous piperacillin-tazobactam $4.5 \mathrm{~g}$ four times daily, vancomycin $1 \mathrm{~g}$ twice daily, and $100 \mathrm{mg}$ oral doxycycline twice daily with oxygen therapy, broncho-dilators, and chest physiotherapy. Fresh frozen plasma was also used for the treatment of DIC.

The patient developed severe tachypnea, hemoptysis, epistaxis, and hypoxia on the second day of hospitalization. The chest $x$-ray showed multiple interstitial and nodular infiltrates with mild bilateral pleural effusions and multiple pulmonary cavities (Figure $1 \mathrm{~b}$ ). These findings were soon confirmed by an urgent computed tomography (CT) chest scan (Figure 1c). Transthoracic and transesophageal echocardiographs excluded infectious endocarditis.
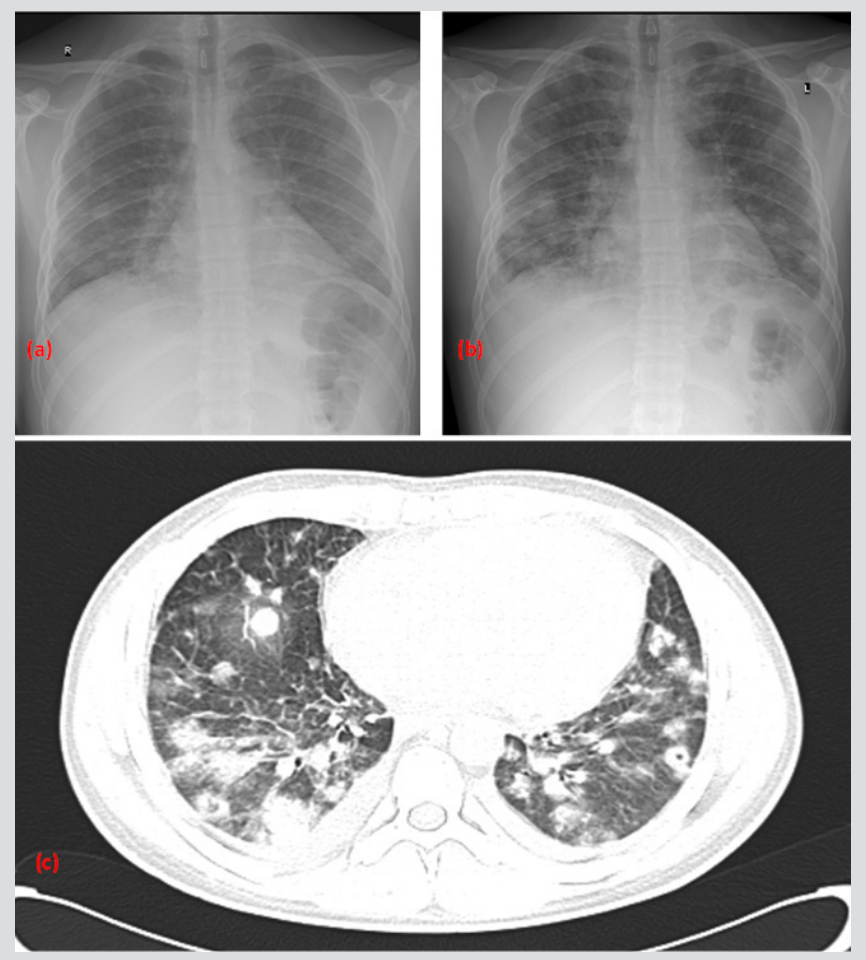

Figure 1: Chest radiograph on Day-1 (Figure 1a), chest radiograph (Figure 1b), and CTChest scan (Figure 1c) on Day-3 of hospitalization showing radiographic deterioration with multiple patchy infiltrates, cavities, and mild pleural effusions.
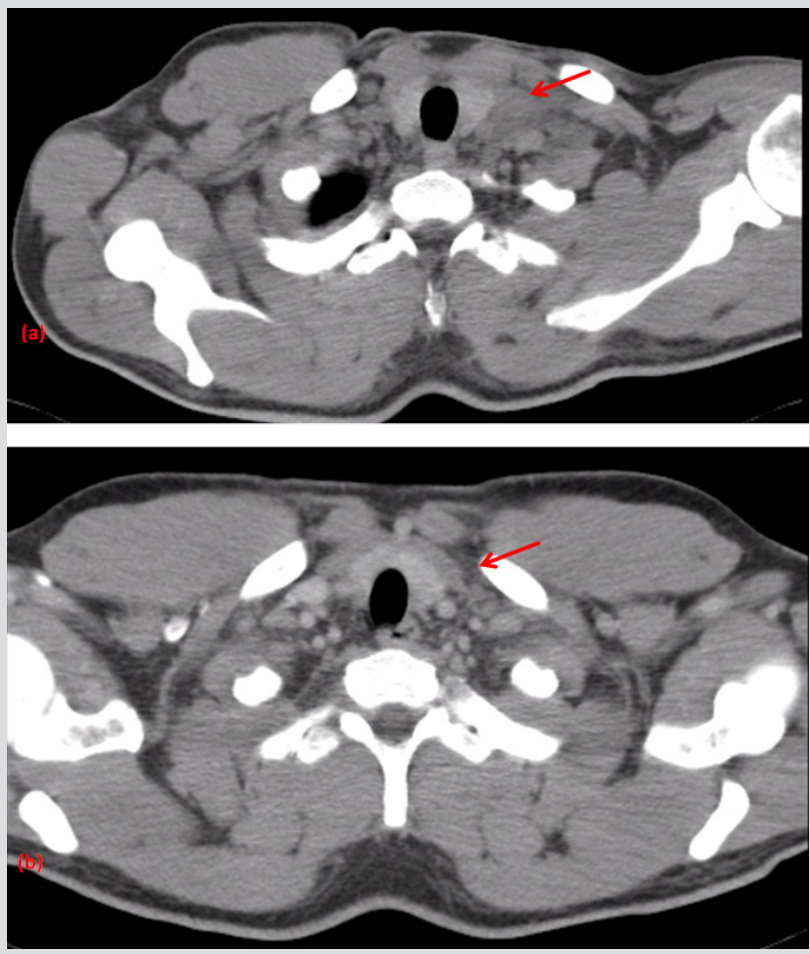

Figure 2: Patient's CT-neck venography showing a mild neck lymphadenopathy and a left complete internal jugular vein thrombosis (red arrow) with extension in the left anonymous vein (Figure 2a) without any resolution of the thrombosis in the CT-neck venography at three month follow-up (Figure $2 b$ ).

Further imaging with Doppler ultrasound (US) of the neck and later a CT-neck venography revealed mild neck lymphadenopathy and left internal jugular vein thrombosis with extension in the left anonymous vein (Figure 2a). So the diagnosis of complicated suppurative (septic) thrombophlebitis of the jugular vein or Lemierre syndrome (LS) was made. Low molecular weight heparin (LMWH) was administered in a therapeutic dose (enoxaparin 8000 IU twice daily subcutaneously) after consulting a vascular-surgeon specialist.

Blood and pleuritic fluid cultures tested positive for Streptococcus gordonii. In addition, rickettsial IgM antibodies were positive for Rickettsia typhi and Rickettsia conorii with 4-fold increase of their titer after two weeks (Table 1b). The antimicrobial therapy was then de-escalated to intravenous penicillin-G 6 million units four times daily and $100 \mathrm{mg}$ oral doxycycline twice daily.

Percutaneous thoracic drainage and a CT-guided transcutaneous catheter drainage were respectively performed (Figure $3 a) 14$ and 18 days after admission due to an increase of the left pleural fluid and the presence of an encapsulated fluid collection in serial chest radiographs. Repeating follow-up fluid culture after drainage was negative for any pathogen.

Further investigation by viral and immunological tests, influenza serology, urine and tonsil swab cultures, tuberculin skin test, streptococcus/ legionella urine antigen, and thrombophilia screening (protein C, protein S, antithrombin III, resistance to protein C) were normal.

Lupus anticoagulant (LA) was positive (LA1/LA2 $=1.3$, reference values $0.8-1.2$ ), but it tested negative when repeated after six weeks. 
Genetic thrombophilia testing revealed three minor mutations with the patient being heterozygous for beta-fibrinogen-455, plasminogen activator inhibitor-1 (PAI-1 4G/5G), and Methylenetetrahydrofolate reductase (MTHFR C677T).

The patient was discharged after 36 days of hospitalization and after completing a month of intravenous penicillin-G and oral doxycycline. He was advised to continue oral antibiotic treatment with $500 \mathrm{mg}$ phenoxypenicillin 4 times daily, and 100 mg doxycycline twice daily, and anticoagulation treatment with subcutaneous enoxaparine 8000 IU twice daily. The 3-month follow-up CT-chest scan showed complete resolution of the pulmonary cavities, infiltrates, and effusions (Figure $3 b$ ) and so the antimicrobial therapy was discontinued. On the contrary, the left jugular vein remained occluded in the follow-up CT-venography (Figure $2 b$ ) and the patient was referred to a hematologist while being prescribed oral anticoagulants with vitamin-K antagonists. He was also referred to a vascular surgeon for possible surgical reconstruction of the obstructed jugular vein and to a dentist for his decayed teeth which were considered as a possible primary infection site.

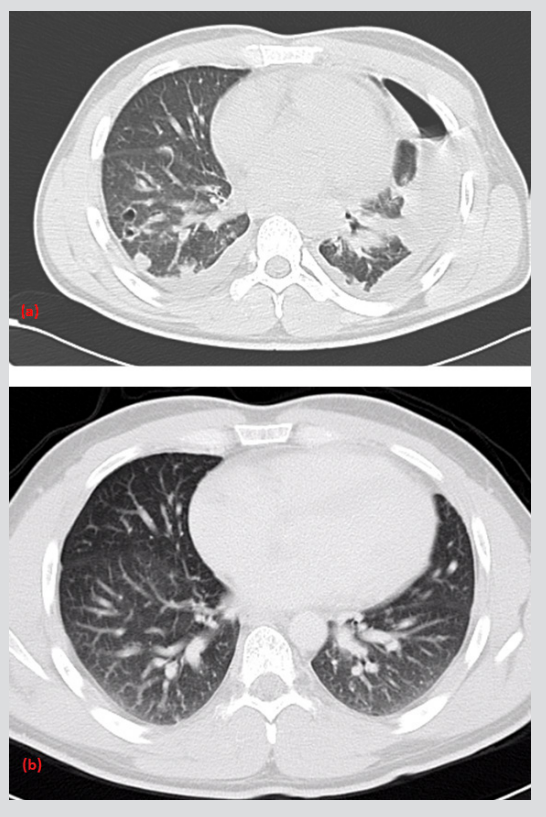

Figure 3. Patient's CT-Chest scan after transcutaneous thoracic catheter and a drainage tube placement on Day-20 showing pulmonary cavities, an organized pleuritic fluid collection, and an iatrogenic pneumothorax (Figure 3a). The CT-Chest scan at 3 months follow-up with complete resolution of the effusions, cavities, and infiltrates of the lungs (Figure $3 b$ )

\section{DISCUSSION}

Lemierre syndrome (LS) or "anaerobic postanginal sepsis" is a septic thrombophlebitis of the internal jugular vein with an incidence of one case in a million annually. After the medical advances leading to its early diagnosis and treatment, the syndrome's mortality rate decreased from $90 \%$ to $15 \%$. There are two categories of LS. The first occurs in young previously healthy people with recent upper respiratory or oral-pharyngeal infections. The second is developed in older adults with predisposition factors and portals of entry distal from the head i.e. central venous access devices, malignancies of the lung, colon, breast, and ovary in addition to thrombophilia ${ }^{[1]}$.

Its main causative agent (81\%) is Fusobacterium necrophorum while $11 \%$ are caused by other Fusobacterium spp. Other causative agents are MRSA, Klebsiella pneumoniae, Viridans streptococci group, Bacteroides fragilis, Peptostreptococcus spp., and Prevotella. Approximately one third of the patients may have poly-microbial bacteremia ${ }^{[2]}$. This case report describes an atypical complicated case of the syndrome in which Streptococcus gordonii and Rickettsiae were isolated and a minor genetic thrombophilic predisposition factors was also present.

Diagnosis isfacilitated after highclinical suspicion in patients with severesepsis, ipsilateral necktenderness parallel to the sternocleidomastoid muscle, and pulmonary compromise (with metastatic abscesses) after an acute pharyngotonsillar infection. The characteristic unilateral suppurative thrombophlebitis of the internal jugular vein is often misdiagnosed as cervical lymphadenitis. The differential diagnosis includes fever, rickettsial infections, endocarditis, tuberculosis, pneumonia, atypical pneumonia such as Legionnaire's disease, and leptospirosis in patients with abnormal liver function tests. The diagnostic approach is thoroughly described in the case description ${ }^{[1]}$.

In this case, blood and pleuritic fluid cultures isolated S. gordonii whereas the serological evaluation showed an increased titer of R. typhi and $R$. conorii IgM. Diagnosis was based on using GenBio IMMUNODOT semi-quantitative enzyme immunoassay for $R$. typhi and $R$. conorii as screening method and was confirmed using a Rickettsia IFA IgM/IgG antibody kit (with Indirect Fluorescence Immunoassay). 
Confirmation PCR of the serum and the pleural fluid was not available at the hospital where the patient was hospitalised. It is interesting that an association between Rickettsiae and S. gordonii infection with LS has never been reported before.

The patient's poor oral hygiene may serve as the S. gordonii entry site to the bloodstream. On the other hand, the epidemiological history of the patient's recent journey from Romania to Cyprus supported possible Rickettsia co-infection. According to the latest literature, $R$. conorii is one of the main pathogens of spotted fever in Romania ${ }^{[2]}$. The patient also presented a 4-fold increase of rickettsial antibodies titer in two sequential plasma samples taken 2 weeks apart, making the diagnosis of the infection definite ${ }^{[3]}$. There is no evidence in the current literature regarding a cross-reaction of Streptococcus spp. causing a false-positive Rickettsia IgM titer. How S. gordonii and R. conorii interacted resulting in LS in this case is not known. Consequently, more research is needed to elucidate the interaction between these two or any other microorganisms in the genesis of LS.

The patient was also screened for hereditary and acquired causes of thrombophilia revealing the minor mutations listed in the case description. There is little in the literature regarding genetically predisposition for developing LS from an oropharyngeal infection.

According to a review, a female patient with LS and genetic polymorphisms presented functional variations of Toll-like-5 receptor (TLR5) gene and two coagulation variations (Tissue Factor (TF) - 603 and Plasminogen-Activator-Inhibitor-1 (PAI-1) 4G-4G homozygous), which are frequently associated with thrombotic events. Another LS case revealed Prothrombin A20210G gene mutation, MTHFR gene polymorphism (C677T) mutation, and increased apolipoprotein levels with normal homocysteine levels ${ }^{[1-4]}$. Screening for an underlying malignancy or thrombophilia should be considered in some cases since an underlying hypercoagulable state may predispose to thrombosis in LS and may alter the type and duration of treatment ${ }^{[4]}$.

The mainstay of LS treatment relies on a combination of broad-spectrum intravenous antibiotics directed against anaerobes, staphylococci, and streptococci (the treatment of choice is b-lactams/beta-lactamase inhibitor antibiotics with or without metronidazol or clindamycin). The optimal duration is uncertain but at least 2-4 weeks of intravenous antibiotics followed by another 2-4 weeks of oral antibiotics is advised. The patient may remain febrile for a prolonged period, which is probably explained by the endovascular nature of the infection and the frequent occurrence of metastatic necrotic abscesses. Surgical drainage of empyema and abscesses is often needed, and open thoracotomies and mediastinal exploration is required in cases of mediastinitis ${ }^{[1]}$.

Furthermore, the literature describes a similar response to treatment with antibiotic and anticoagulation versus antibiotic therapy alone ${ }^{[5]}$. Most authors suggest treatment with heparin and antibiotics with discontinuation when acute illness resolves in order to avoid complications. Other researchers recommend anticoagulation in patients with significant clot burden (such as retrograde extension, bilateral neurological features due to endocranial thrombosis) and discontinuation when the infection is controlled and venous re-cannulation has been achieved ${ }^{[5]}$. To conclude, the authors of this article believe that anticoagulation in addition to antibiotic therapy is essential in patients with severe LS and extending thrombus, especially in cases with known thrombophilic disease or newly diagnosed thrombophilia predisposition.

\section{REFERENCES}

1. Riordan T. Human infection with Fusobacterium necrophorum (Necrobasillosis) with a focus on Lemierre syndrome. Clin Microbiol Rev 2007;20:622-659.

2. Hagelskjaer LH, Prag J, Malczynski J, Kristensen JH. Incidence and clinical epidemiology of necrobacillosis, including Lemierre's syndrome, in Denmark 1990-1995. Eur J Clin Microbiol Infect Dis 1998;17:561-5.

3. Walker DH. Rickettsiae and Rickettsial Infections: The Current State of Knowledge. Clin Infect Dis 2007;45:S39-44.

4. Constantin JM, Mira JP, Guerin R, Cayot-Constantin S, Lesens O, Gourdon F et al. Lemierre's syndrome and genetic polymorphisms: a case report. BMC Infect Dis 2006;6:115.

5. Phua CK, Chadachan VM, Acharya R. Lemierre syndrome-should we anticoagulate? A case report and review of the literature. Int J Angiol $2013 ; 22: 137-142$. 